\title{
Les modes extrajudiciaires de règlement des litiges en Côte d'Ivoire
}

\author{
Par Eugène Assi Assepo
}

"Si on osait une métaphore, on pourrait considérer le recours à la justice d'Etat comme la partie émergée d'un iceberg. Bien que visible institutionnellement, peut-être cette justice d'Etat ne représente-t-elle qu'une part infime des modalités de prise en charge et de solution des différends". Cette réflexion du professeur Etienne Leroy ${ }^{1}$, à propos de la justice en Afrique, pour être choquante, n'en est pas moins révélatrice d'une situation: la préférence des justiciables africains pour une autre justice, ou une justice autrement. La raison de cette défiance vis-à-vis de la justice officielle réside essentiellement dans la finalité recherchée par les parties à travers la solution du litige. A cet égard, l'on sait qu'en Afrique, la justice était conçue comme une fonction de conciliation, de négociation, de médiation ${ }^{2}$.

Le droit traditionnel africain avait, en effet, pour objectif la préservation de la cohésion sociale. Cela se percevait à travers la façon dont la justice était rendue: il fallait à tout prix éviter une rupture entre les membres d'un même groupe social. Le Droit avait alors une allure souple faite de compréhension et de recherche du compromis se caractérisant le plus souvent par l'accession à une solution acceptée de tous ${ }^{3}$. Cette situation sera bouleversée avec l'avènement de la colonisation puis l'accession des colonies à l'indépendance. Indépendante, la Côte d'Ivoire va se doter, en matière judiciaire, d'une législation moderne de type occidental. Or, la justice, on le sait, est une manifestation du pouvoir de juger que l'Etat délègue aux juges qu'il institue à cet effet. Dès lors, ce pouvoir apparaît comme une fonction de l'Etat, une attribution de la souveraineté étatique qu'organise la Constitution au

(E.) Leroy, Le justiciable africain et la redécouverte d'une voie négociée de règlement des conflits, Afrique contemporaine, $\mathrm{N}^{\circ} 156$ (spécial), La justice en Afrique, 1990, p. 111. Un auteur a même parlé de "justice ... sans justiciables": (J.) Du Bois du Gaudusson, Le statut de la justice dans les Etats d'Afrique Francophone, Afrique Contemporaine, op. cit, p. 11.

Voir Keba M'baye, Quel avenir pour la justice en Afrique?, Afrique Contemporaine, op. cit., p. 290; (M.) Kamto, Une justice entre tradition et modernité, Afrique contemporaine, op. cit., p. 58; (J.) Assi Benié, Le médiateur ivoirien, Inédit, p. 5; (P.) Nkou Mvondo, La crise de la justice de l'Etat en Afrique noire francophone, Penant 1997, n 824, p. 210; (O.H.) Legre, "L'image du juge dans les sociétés traditionnelles", in Le juge: une figure d'autorité, Paris, l'Harmattan, 1996, p. 75 et s.; (A.) Chaïbou, Le rôle des juges traditionnels dans le processus de règlement des conflits au Niger, in Le juge: une figure d'autorité, ibid., p. 143.

Voir (M.M.) Hachirou, La transaction en matière pénale au Niger, mémoire, DEA, Faculté de Droit Abidjan, 1993-1994, p. 70 et s. 
titre de l'autorité judiciaire. Mais, par son introduction dans l'organisation institutionnelle de la société, la justice acquiert selon le pays considéré, un particularisme plus ou moins marqué, une indépendance plus ou moins grande à l'égard de l'Etat, une spécificité fonctionnelle plus ou moins réduite avec d'autres voies de solution des litiges ${ }^{4}$.

Ainsi, la Côte d'Ivoire, à l'instar des autres Etats africains francophones, voit le fonctionnement de ses institutions judiciaires concurrencé par la permanence de pratiques traditionnelles. Cela se remarque en particulier dans la vie judiciaire où diverses autorités non juridictionnelles (chefs coutumiers, autorités religieuses, politiques ou administratives) exercent, de manière informelle, une importante activité ${ }^{5}$.

Cet état de fait traduit, en réalité, l'inadaptation du cadre de règlement des litiges d'inspiration étrangère au contexte socioculturel ivoirien ${ }^{6}$. La raison de cette situation réside dans le conflit de cultures: la culture des sociétés africaines traditionnelles est marquée par les valeurs sociales collectives alors que la culture occidentale prend en compte les valeurs individuelles. Dès lors "la coexistence ou la superposition des deux cultures dans les Etats africains indépendants renvoie à une opposition des conceptions de la fonction sociale de la justice dans ces pays: opposition entre l'équité et le glaive, entre l'esprit de conciliation et l'esprit de combat" ${ }^{7}$. Ainsi, comme le relève, fort justement, le professeur (R.) DégniSégui "le droit de recourir à la justice se trouve dans son exercice en situation concurrentielle voire conflictuelle permanente avec les droits traditionnels", plus loin l'auteur écrit "à l'envahissement du droit dominant étranger qui, ayant le support étatique, triomphe sur le plan juridique formel, les droits dominés qui, eux, bénéficient de l'appui populaire, opposent de vives résistances".

Voir (B) Oppetit, Justice étatique et justice arbitrale, in Etudes offertes à Pierre Bellet, Litec, 1991, p.415

5 Voir (R.) Degni-Ségui, L'accès à la justice et ses obstacles, in L'effectivité des droits fondamentaux dans les pays de la communauté Francophone, AUPELF-UREF, 1994, p. 249; du même auteur: Modes informels de régulation dans les quartiers urbains pauvres. L'exemple d'Abidjan, inédit, p. 20 et s.; (J.) Assi Benié, op. cit. p. 5; (A.) Mignot, La justice traditionnelle, une justice parallèle. L'exemple du Sud Togo, Penant, 1982, p. 5; (M. M.) Hachrou, op. cit., p 69 et s.; (A.) Chaïbou, op. cit. p. 137 et s.; (U.) Spellenberg, Unité et dualisme dans les systèmes judiciaires en Afrique, in Le juge: une figure d'autorité, op. cit. p. 153 et s. Voir (J.) Assi Benié, op. cit., p. 4 et s.; (P.) Nkou Mvondo, op. cit. p. 209 et s.

(M.) Kamto, op. cit.,p. 58, note 1; voir aussi (P.) Nkou Mvondo, op. cit, p. 209 et s. Cette conception correspond à la doctrine du Professeur Alain Seriaux [Le droit: une introduction, Ellipses, 1997, $\mathrm{N}^{\circ} 171$ ] selon laquelle "il n'existe que deux modes authentiques de règlement des conflits: la restitution et la réconciliation".

(R.) Degni-Ségui, L'accès à la justice et ses obstacles, op. cit., p. 242. 
Ces considérations ne peuvent expliquer à elles seules la faible fréquentation, par les justiciables, des tribunaux étatiques. Elle se justifie aussi par ce qu'il est convenu d'appeler la crise de la justice. Certes, la crise se retrouve dans plusieurs pays ${ }^{9}$, mais elle l'est davantage en Côte d'Ivoire, tout comme dans les autres pays africains francophones.

Selon les conclusions du groupe régional africain de l'Union Internationale de la Magistrature, réuni à Abidjan les 16 et 17 avril 1998, autour du thème "Justice et développement économique en Afrique", "les griefs faits à la justice, notamment par les opérateurs économiques et les bailleurs de fonds, se résument en un sentiment d'insécurité tant juridique que judiciaire. Ainsi reproche-t-on à la justice son caractère partisan et sa lenteur, et aux magistrats leur manque d'indépendance et d'intégrité" ${ }^{10}$. Ce constat résume assez bien les défauts majeurs de la justice étatique ivoirienne. Ces maux constituent autant d'obstacles à l'accès au prétoire. Des auteurs ivoiriens ${ }^{11}$ ou africains ${ }^{12}$ les ont très bien stigmatisés. Parmi ces imperfections, on peut relever l'éloignement du justiciable (des tribunaux) ${ }^{13}$, les lenteurs de la procédure et sa trop grande complexité ${ }^{14}$, le coût de la justice ${ }^{15}$, la dépendance des juges ${ }^{16}$, la langue et le langage des tribunaux ${ }^{17}$ et le système politique ivoirien ${ }^{18}$.

Sur la crise de la justice civile en France, voir (L.) Cadiet, Droit judiciaire privé, Paris, Litec, 2è éd., $1998 \mathrm{n}^{\circ}$ 71; (P.) Godé, Le mensonge dans le procès civil, in Etudes dédiées à Alex Weill, Dalloz Litec 1983, p. 259 et s., voir Spéc. p. 277 n 21 où l'auteur relève que: "Asphyxiée par une surcharge de labeur quotidien, la justice se dirige à vue, acceptant de rentrer dans une logique de rentabilité quantitative".

10

11

12

13

14

15

16

17

18 
$\mathrm{Au}$ nombre des remèdes préconisés à la crise de la justice, on a proposé sa privatisation ${ }^{19}$. Mais les regards se sont, en général, plutôt dirigés vers une autre direction: le développement des substituts ou des alternatifs à la justice étatique. Le phénomène est d'actualité dans plusieurs pays du monde: c'est la déjudiciarisation qui se traduit par l'émergence ou le développement des modes alternatifs de règlement des conflits. De fait, à travers des études, enquêtes, colloques, rapports, monographies, débats, les modes alternatifs de règlement des litiges apparaissent, depuis quelques années, comme l'une des questions les plus discutées, lorsque l'on s'interroge sur l'évolution contemporaine du droit ${ }^{20}$. La question présente le mérite de fournir le cadre d'un vaste débat sur la place du juge et du droit dans le règlement des conflits ${ }^{21}$. De fait, la résolution du litige se fait, généralement, par le recours à un tiers; or "dans tous les systèmes de droit, ce recours à un tiers est le fondement même du fonctionnement de la justice" 22 .

En Côte d'Ivoire, ce tiers prend souvent la forme d'un conciliateur. C'est pourquoi, avec l'intervention de la loi du 9 août 1993 relative à l'arbitrage, la création puis l'installation de la Cour d'arbitrage de Côte d'Ivoire (CACI) le 12 août 1997, la création de la Cour commune de justice et d'arbitrage de l'Organisation pour l'harmonisation du droit des affaires en Afrique (OHADA) et la mise en place d'un Organe Présidentiel de Médiation

répondre à ce réel besoin que le président de la république a crée l'OPREM [l'organe présidentiel de médiation]": (J.) Assi Bénié, Le médiateur ivoirien, op cit., p.6.

19

20 Justices, $\mathrm{n}^{\circ} 1$, janv.-juin 1995 , p. 135 et $\mathrm{s}$.

Voir: Les modes alternatifs de règlement des conflits (rencontres internationales de droit comparé, Damas, 5-8 octobre 1996), Revue internationale de droit comparé (RIDC) 2, 1997, p. 313 et s.; (J.F.) Guillemin, Les nouvelles attentes des entreprises en matière de règlement des conflits, Revue de l'arbitrage, 1996, n 4, p. 583 et s. (M.C.) Rivier, Les modes alternatifs de règlement des conflits en droit du travail, Justices, $\mathrm{n}^{\circ}$ 8, oct.-déc. 1997, p.33 et s.; (B.) Oppetit, Les modes alternatifs de règlement des différends de la vie économique, Justices, ${ }^{\circ} 1$, janv.-juin 1995, p. 53 et s.; Les modes non judiciaires de règlement des conflits (actes de la journée d'études du 27 avril 1994, sous la direction de l'Association Européenne pour le droit bancaire et financier (AEDBFBelgium) et l'Association Belge des juristes d'entreprise (ABJE), Bruxelles Bruylant 1995; (L.) Cadiet, Solution judiciaire et règlement amiable des litiges: de la contradiction à la conciliation, in Mélanges Claude Champaud, Dalloz, 1997, p.123 et s.; du même auteur, Le spectre de la société contentieuse in Mélanges (G.) Cornu; PUF, 1994 p. 29 et s.; (P.) Kayser, La recherche en France de la diminution du contentieux judiciaire et administratif par le développement des règlements amiables, Justices, $\mathrm{n}^{\circ}$ 3, janv.-juin 1996, p.203 et s.; (J.-M.) Mousseron, "responsable mais pas coupable" in Mélanges Christian Mouly, Litec, 1998, T. II, p. 141 et s.; (J. F.) Overstake, Cours de droit judiciaire privé, licence 1997-1998, Librairie Montaigne, Bordeaux, p. 24 et s.
(M.) Chemillier-Gendreau, Introduction générale au droit, Paris, éd. Eyrolles, 1990 p. 11 
(OPREM) le 29 septembre 1995, il nous semble utile de faire le point sur les modes extrajudiciaires ${ }^{23}$ de règlement des litiges en Côte d'Ivoire.

Il apparaît ainsi, que face à la crise de la justice moderne, la réaction de la société globale a consisté à favoriser le retour en force de la voie négociée de règlement des conflits ${ }^{24}$, concrétisé par la persistance des modes traditionnels de règlement des litiges (I) et l'avènement de modes nouveaux de règlement des litiges (II).

\section{La persistance des modes traditionnels de règlement des litiges}

Le rejet du juge moderne va amener le justiciable ivoirien à rechercher d'autres modes de solution de litiges ; parmi ceux-ci, un procédé ancestral tient une bonne place: la conciliation. Celle-ci n'est qu'un ensemble de concessions plus ou moins réciproques que se font les parties. "contractuellement formalisées, ces conciliations sont dénommées transactions par les juristes" ${ }^{25}$.

Transaction et conciliation sont donc liées pour constituer les modes ancestraux de résolution des litiges. Dans le contexte ivoirien, la transaction a un visage formel (A), alors que la conciliation se présente, généralement, comme une solution informelle (B).

\section{A. Un mode formel: la transaction}

Accord des parties sur la solution du litige, la transaction sera utile aux pouvoirs publics pour la régulation de la justice. Cette solution revêt un double aspect: le maintien, en matière civile, de la solution classique de la transaction ; et l'extension de son domaine en matière pénale.

\section{Le maintien de la solution classique en matière de transaction civile}

La transaction n'a pas fait, en Côte d'Ivoire, l'objet d'une réglementation particulière, en matière civile. Ce sont, en effet, les dispositions du code civil français qui y sont toujours

23

Le terme s'étend de toute procédure ou procédé qui a lieu en dehors d'une instance en justice. L'expression "amiable" aurait pu être retenu, mais nous l'avons écarté car, soit (au sens strict) il ne prend pas en compte l'arbitrage, soit (au sens large) il prend également en compte un accord obtenu, entre les parties, devant le juge, ce qui est hors de notre étude.

24

25

Voir (E.) Leroy, Le justiciable africain et la redécouverte d'une voie négociée de règlement des conflits, op cit., p. 114. 
applicables. La transaction est ainsi régie par les articles 2044 à 2058 du code civil. L'article 2044 la présente comme un "contrat par lequel les parties terminent une contestation née, ou préviennent une contestation à naître". Cette définition est maladroite ${ }^{26}$. La transaction met, en effet toujours fin à un litige né; seulement, il n'est pas nécessaire qu'un juge ait déjà été saisi. Par ailleurs, la disposition de l'article 2044 du code civil ne rend pas compte de la nature spécifique de la transaction qui est de reposer sur des concessions réciproques des parties ${ }^{27}$ et d'emporter renonciation mutuelle de leur droit d'action: il y a action dans transaction; plus que la volonté, même commune, de mettre fin au litige, c'est la renonciation à l'action qui est la marque propre de la transaction ${ }^{28}$. L'effet principal de la transaction écrivent les professeurs Malaurie et Aynes est d'éteindre l'action en justice relative au droit litigieux. Désormais, aucun procès ne pourra plus être engagé; s'il y en avait un en cours, il s'achève par le dessaisissement du juge: il n'y a plus rien à juger ${ }^{29}$. On a pu alors en déduire qu' « elle se rapproche, ainsi, de l'obligation de ne pas agir en justice qui a pour conséquence d'interdire toute saisine d'une juridiction étatique ou arbitrale" ${ }^{30}$.

La transaction présente donc des avantages majeurs: simplicité, rapidité, économie financière ; c'est pourquoi on a coutume de dire "qu'une mauvaise transaction vaut mieux qu'un bon procès" ${ }^{31}$. Ce mode contractuel de règlement des litiges ${ }^{32}$ s'inscrit ainsi dans la droite ligne de la recherche des solutions des différends en dehors du juge. On doit cependant se demander quelle est la force intrinsèque de cette solution conventionnelle surtout qu'elle a pour objectif de remplacer une solution juridictionnelle. A cet égard, l'article $2052 \mathrm{al} .1 \mathrm{du}$ code civil dispose: "les transactions ont, entre les parties, l'autorité de la chose jugée en dernier ressort". Le caractère surprenant ou encore la fausseté de cette expression a maintes fois été dénoncée, notamment en ce qu'il est fait référence à l'autorité de la chose jugée en dernier ressort ${ }^{33}$.

(L.) Cadiet, Droit judiciaire privé, op cit., $\mathrm{n}^{\circ} 941$.

(L.) Cadiet, ibid; voir égal: (G.) Cornu et (J.) Foyer, Procédure civile, PUF / Thémis, 3è dé, 1996, $\mathrm{n}^{\circ} 8$, (P.) Kayser, "La recherche en France de la diminution des contentieux judiciaire et administratif par le développement des règlements amiables, Justices, $n^{\circ} 3$ janv-juin 1996, p. 203 et s., voir spéc. p. 212; (Ch.) Jarrosson, La notion d’arbitrage, L.G.D.J., Paris, 1987, n 398.

(L.) Cadiet, ibid. Bien entendu, le recours à ce mode de règlement suppose que les parties aient la libre disposition de leurs droits et qu'elles respectent l'ordre public.

(Ph.) Grignon: L'obligation de ne pas agir en justice, in Mélanges Christianes Mouly (T. II), Litec, 1998, p. 115 et s., voir Spéc. p. 122, n 11. (J.-P.) Scarano: Institutions Juridictionnelles, Ellipses, / Editions Marketing S.A., 1996, p. 27, $\mathrm{n}^{\circ}$ 32.

Voir à ce sujet, (J.M.) Mousseron: Technique Contractuelle, Edition Juridique Lefevre, 1998, $\mathrm{n}^{\circ}$ 1452 et s. L'auteur y présente les clauses tendant à écarter le traitement judiciaire des litiges et, parmi celles-ci, les clauses tendant à favoriser la transaction.

(L.) Cadiet, Droit Judiciaire Privé, op cit., Litec 1998, n 944. 
C'est ainsi que le professeur Francis Kernaleguen ${ }^{34}$ a pu se demander s'il fallait en conclure que parce que la transaction atteint le même résultat qu'une décision de justice, elle participe de son autorité spécifique. Pour M. Kernaleguen une réponse négative s'impose. La meilleure preuve en est que lorsqu'une des parties veut attaquer la transaction, elle n'exerce pas une voie de recours comme elle le ferait pour une décision dotée de l'autorité de la chose jugée, elle intente une action en nullité. En réalité, dit cet auteur, la formulation malheureuse de l'article 2052 tend simplement à mettre en relief la similitude de fonctions entre les transactions et les jugements ${ }^{35}$; elle exprime l'effet extinctif attaché à cette convention. De fait, cet effet présente le même caractère relatif que l'autorité de la chose jugée (art. 1351 du code civil). Il est limité quant à l'objet et quant à la cause (art. 2048 et 2049 du code civil) et même quant aux personnes (art. 2050). Mais la relation de cette relativité, affirme le professeur Kernaleguen, s'établit tout autant avec l'article 1165 du code civil concernant l'effet relatif des conventions. Cet effet extinctif aura pour conséquence que si l'une des parties à la transaction prétend par la suite soumettre au juge une action touchant à la contestation éteinte, l'autre partie pourra s'y opposer en invoquant une fin de non recevoir tirée de l'existence de la transaction. Pour établir son droit d'action contesté, le demandeur devra soit démontrer que sa prétention n'est pas comprise dans l'objet de la transaction qui lui est opposée, soit poursuivre l'annulation de la transaction ${ }^{36}$ (annulation qui ne peut être poursuivie ni pour erreur de droit ni pour lésion, cf. art. $2052 \mathrm{c}$. civ). Ainsi envisagée, la transaction ne concerne que la matière civile. Elle éteint l'action civile. Le droit criminel, plus particulièrement la procédure pénale ne fait pas une aussi large part à la transaction. Cela s'explique par le caractère d'ordre public de l'action publique $^{37}$.

(F.) Kernaleguen: La solution conventionnelle des litiges in (L.) Cadiet (travaux coordonnés par) Le droit contemporain des contrats. Travaux et Recherches de la Faculté de Rennes. Ed. Economica 1987, p.65 et s., voir Spéc. p. 81 et 82.

Voir en ce sens, (P.) Kayser, op cit., p. 213: on peut expliquer la formule par une assimilation de la force obligatoire des contrats à l'autorité de la chose jugée.

Un autre problème relatif à la transaction a trait à son caractère translatif ou déclaratif: Si la doctrine la plus récente reconnaît à la transaction un caractère déclaratif [voir p. ex: (P.) Kayser, Ibid.] elle ne conférerait pas aux parties des droits nouveaux mais aurait seulement pour effet de reconnaître et de consolider les droits qu'elles ont ou prétendent avoir; cette position ne fait cependant pas l'unanimité: un courant doctrinal défend la conception de l'effet translatif de la transaction qui devrait être considérée comme une cession de droit litigieux entre parties [voir $(L$.) Cadiet, Droit judiciaire privé op cit., $\mathrm{n}^{\circ} 945$ et note 353 ; voir aussi sur la controverse: (G.) Cornu et (J.) Foyer, Procédure Civile, PUF / Thémis, 3è éd., 1996, n 8, p. 47 et 48].

Voir (B.) Adjé, La transaction pénale: exemple des transactions douanières et fiscales, mémoire, DEA Faculté de droit Abidjan, 1987-1988, p. 4; voir aussi en droit Nigérien: (M. M.) Hachirou, op. cit. Voir égal. (M.) Dobkine, La transaction en matière pénale, D. 1994, op. cit. p. 137. 


\section{L'extension du domaine de la transaction en matière pénale}

Le ministère public, bien que jugeant de l'opportunité de l'exercice de l'action publique, ne peut en disposer à sa guise. Et pourtant l'article 6, alinéa 3 du code de procédure pénale ivoirien prévoit la transaction comme l'une des causes d'extinction de l'action publique. Le champ de la transaction pénale est donc nécessairement réduit et la transaction pénale ne peut être conclue que par les ministères publics spécialisés que constituent certaines administrations chargées de protéger certains intérêts particuliers (essentiellement pécuniaires) de l'Etat. C'est le cas dans des matières spécifiques telles que la douane, les impôts, les eaux et forêts. La loi permet à ces administrations de transiger avec le ou les contrevenants afin de terminer totalement l'affaire.

Cette solution est très avantageuse à la fois pour le délinquant qui échappe à la rigueur des lois pénales et pour l'administration qui fait l'économie d'un procès et les suites que celui ci peut entraîner ${ }^{38}$. La transaction exigeant que les parties se fassent des concessions réciproques, on peut se demander sur la base de quel(s) accord(s) cet arrangement peut intervenir. De la part de l'administration, les concessions sont révélées par l'abandon des poursuites. Le contrevenant lui, consent à payer les pénalités transactionnelles. Celles-ci sont d'ordinaire fixées en argent. Elles peuvent s'exécuter parfois en nature c'est le cas notamment lorsque le délinquant fait abandon à l'administration des douanes, des marchandises et des moyens de transport saisis. La récente loi du 23 décembre $1998^{39}$ vient d'étendre le champ d'application de la transaction pénale à d'autres types d'infractions ${ }^{40}$.

Quelles sont les raisons justifiant cette extension? Il ressort de l'exposé des motifs de la loi nouvelle que celle-ci vise à apporter un début de solution à la lenteur de la justice ivoirienne à statuer sur les causes qui lui sont soumises; lenteur due essentiellement à l'engorgement des juridictions et des greffes. De fait, on note dans l'exposé des motifs que la transaction présente de nombreux avantages:

Voir (B.) Adjé, ibid.; (M.) Dobkine, op cit.

39 Loi n ${ }^{\circ}$ 98-745 (J.O.R.C.I., n 5 du 4 février 1999, p. 67 et s.) Cette loi instaure également l'acquiescement qui consistera, pour les parties privées à un procès pénal, à marquer leur accord au jugement rendu, avant l'expiration des délais d'appel: le prévenu approuve la condamnation et s'engage à payer l'amende qui lui a été infligée, ainsi que les dommages intérêts alloués à la victime, et que celle-ci a acceptées [article 455 nouveau C.P.P.]. Cette procédure, relève-t-on dans l'exposé des motifs, devrait permettre de réduire sensiblement le nombre d'appels. Elle est rendue initiative par l'échéancier de paiement ou la réduction de l'amende de 50\%, qui peuvent être accordés au condamné qui acquiesce au jugement.

40

La transaction exclut les crimes pour ne concerner que les contraventions et les délits, à l'exception de ceux qui troublent gravement l'ordre public, notamment les infractions commises sur les mineurs ou les personnes incapables de se protéger, les détournements de deniers publics, les attentats aux mœurs etc:. article 8 nouveau C.P.P. 
- elle permettra de désengorger les formations de jugement en les délestant des affaires de moindre importance qui, sans perturber gravement l'ordre public, doivent être poursuivies, pour éviter que l'inertie de la justice n'encourage la délinquance;

- elle évitera les suspicions, susceptibilités et frustrations que peut générer une décision de classement sans suite;

- le parquet fait l'économie d'un dossier, le délinquant évite un procès pénal et la victime peut être rapidement désintéressée.

Tels sont les arguments avancés en faveur de la réforme. A l'analyse, on peut se demander si d'autres raisons ne peuvent pas expliquer cette nouvelle orientation. On peut, notamment, se demander si cette loi ne vient pas consacrer une pratique: la pratique d'une transaction en matière pénale de caractère informel. A cet égard, on peut citer la pratique judiciaire dans le cadre de l'action publique (dont le ministère public détient le monopole) permettant d'aboutir à une décision de classement sans suite. D'ailleurs cette situation a été prise en compte par les initiateurs de la loi.

Mais il y a aussi et surtout la pratique policière, en raison de la tendance des autorités policières à s'ériger en médiateurs des parties ${ }^{41}$. De fait, généralement, lorsque la victime d'une infraction saisit la police, elle fait expressément mention à l'autorité policière que le but de sa plainte n'est pas l'engagement d'un procès. Il s'agit plutôt d'intimider le débiteur pour l'amener à s'exécuter plus promptement; du moins pour ce qui est de certaines infractions. Ainsi, par exemple, en matière d'abus de confiance, à partir du moment où le délinquant reconnaît l'infraction dès sa comparution devant les autorités de police, la victime s'attend à juste titre, à le voir contraindre à s'exécuter en sa faveur. Cela n'est possible que par la voie de la transaction ${ }^{42}$. En effet, dans le cadre de cette dernière, une affaire se règle en une journée à la police ou au parquet. Lorsque la voie contentieuse est utilisée, le fait de mettre le délinquant en prison ne facilitera pas l'exécution de son obligation. Où trouver l'argent nécessaire pour honorer sa dette? La condamnation, par le juge répressif, du délinquant à des dommages - intérêts aura en réalité peu d'effets. En revanche, lorsque la victime accepte de transiger, même si le délinquant n'est pas solvable, ses parents peuvent

Voir à cet égard en droit Nigérien [mais la situation dans ce pays africain n'est pas différente de celle de la Côte d'Ivoire], (M. M.) Hachirou, La transaction en matière pénale au Niger,op cit. p. 69 et s. Voir aussi à titre de comparaison, et en droit français, (Ch.) Lazerges, Typologie des procédures de médiation pénale, in Mélanges offerts à (A.) Colomer, Litec, 1993, p. 217 et s.: L'auteur y parle du gendarme conciliateur ou médiateur à l'occasion de la commission d'un petit délit, et de manière plus générale, dans le cadre de ce qu'elle dénomme la médiation pénale sociétale sans contrôle judiciaire, elle écrit: "pour la médiation sociétale individuelle et informelle, il suffit de préciser qu'elle est celle du citoyen exercée de façon informelle dans le cadre de sa vie familiale professionnelle, sociale; pensons aux médiations de ce type effectuées par des gardiens de la paix, des mères de famille, des prêtres, des pasteurs, des enseignants". 
rembourser la somme qu'il doit. Ces effets de la transaction se retrouvent également dans la conciliation.

\section{B. Un mode informel: la conciliation}

La conciliation est définie comme l'accord par lequel deux personnes en litige mettent fin à celui-ci (soit par transaction, soit par abandon unilatéral ou réciproque de toute prétention), la solution du différend résultant non d'une décision de justice ni même de celle d'un arbitre mais de l'accord des parties elles-mêmes ${ }^{43}$. L'usage de la conciliation comme mode de règlement des conflits est un thème permanent dans toute réflexion sur la justice ${ }^{44}$. C'est que dans son sens strict, la conciliation est un mode de solution amiable du litige entre particuliers ${ }^{45}$. Ce procédé doit, à cet égard, être distingué de la médiation. En effet, les deux notions, quoique voisines, ne sont pas synonymes, encore que la pratique ne les distingue pas toujours très nettement ${ }^{46}$. C'est qu'il semble difficile de distinguer ces deux modes. L'un et l'autre comportent l'intervention d'un tiers, neutre et indépendant qui tente de rapprocher les parties en litige, d'explorer les solutions possibles et d'amener les parties à y consentir. Selon certains auteurs ${ }^{47}$, la médiation ne serait qu'une variété de conciliation; le médiateur pouvant mettre au point et suggérer lui-même aux parties une formule d'accord, alors que le conciliateur aurait un rôle plus passif se limitant à informer les parties de leurs situations, de leurs intérêts, de leurs droits et obligations respectifs, à les rapprocher, à les faire communiquer entre elles et à tenter d'obtenir leur adhésion à une solution commune qui serait ensuite consignée dans un accord ou une transaction ${ }^{48}$.

Quoi qu'il en soit, dans le cas précis de la Côte d'Ivoire, étant donné qu'un Grand Médiateur a été nommé ces dernières années ${ }^{49}$ et que les autorités villageoises restent de facto

(G.) Cornu, Vocabulaire judiciaire, P.U.F., 7è éd., 1998, p. 181.

(J.) Van Compernolle, Le juge et la conciliation en droit judiciaire belge, in Mélanges en l'honneur de Roger Perrot, Dalloz, 1996, p 523.

(L.) Cadiet, Le spectre de la société contentieuse, p. 44, $\mathrm{n}^{\circ} 25$.

(B.) Oppetit, Les modes alternatifs de règlement des différends de la vie économique, In Justices, noir $n^{\circ} 1 /$ janv.-juin 1995, p. 53 et s., $V^{\circ}$ spéc. p. 56. Un auteur emploie même simultanément les deux termes: "l'arbre à palabre "symbole de la médiation-conciliation, peut-il, doit-il et sous quelles formes s'imposer comme un mode de régulation des conflits et litiges". (Ch.) Lazerges, op cit. 1993 , p. 217.

Voir par exemple, (Ch.) Jarrosson n ${ }^{\circ}$ 348. L'auteur écrit: "la médiation n'est qu'une forme de conciliation opérée par un tiers, qui elle-même n'est qu'une forme de conciliation lato sensu."

(J.) El-Hakim, Les modes alternatifs de règlement des conflits dans le droit des contrats, in: Les modes alternatifs de règlement des conflits, Revue Internationale de droit comparé, 2, 1997, p. 347 et s. Voir. p. 349.

Voir Infra, p 25 et s. 
"investies" du pouvoir de régler pacifiquement les litiges survenant au sein de leurs communautés, nous retenons la distinction médiation / conciliation; les autorités susvisées étant concernées par la deuxième voie de règlement pacifique des différends. La propension des autorités à accepter les modes extrajudiciaires réside dans ce que le professeur Lazerges ${ }^{50}$ nomme "l'ordre négocié", ordre qui prédomine dans les sociétés traditionnelles et qui leur permet de se prémunir de la division sociale. Il faut, pour comprendre cette situation, interroger l'histoire, puis voir la survivance de la fonction judiciaire des autorités traditionnelles avant de terminer avec la justice de proximité.

\section{L'existence séculaire de la conciliation}

La conciliation a toujours existé dans les sociétés africaines; et l'image de "l'arbre à palabre", arbre sous lequel les chefs traditionnels se réunissent avec leurs sujets pour prendre des décisions importantes pour la communauté, mais aussi, pour trancher - pacifiquement - les litiges est bien connue ${ }^{51}$. La Côte d'Ivoire s'inscrit dans cette perspective qui permet d'entrevoir la conciliation avant et pendant la colonisation. Au cours de la période précoloniale, les chefs traditionnels sont tributaires de pouvoirs de conciliation en matière de règlement de litiges ${ }^{52}$. C'est ainsi, que dans la région de Séguéla, les palabres ne sont soumis à aucun protocole ni lieu d'élection particulier. C'est une simple assemblée de famille où tout le monde donne son avis. La juridiction varie avec l'importance de la cause; il n'y a pas de juge, celui qui préside l'assemblée est l'individu le plus âgé d'une famille tant que ses facultés ne sont pas trop faibles ${ }^{53}$.

Si deux individus de la même famille ont entre eux une affaire de dette ou de règlement de comptes, ils s'adressent à l'individu le plus âgé de la famille, chacune des parties est soutenue par ses parents; le chef de famille prend les avis des individus présents, donne le sien et à force de conseils, de discours, amène les individus à tomber d'accord. Il n'y a pas de

(Ch.) Lazerges, op cit. p. 217 et s. Voir Spéc. p. 233 et 234 l'auteur y oppose à la notion "d'ordre négocié" celle "d'ordre juridique étatique imposé".

Voir (M.) Kamto, op cit. p. 57 et s.; (M.) Debène, La justice sans juge, d'hier à demain, Afrique contemporaine, op cit., p. 86 et 87. (Ch.) Jarrosson [la notion d'arbitrage, op cit., 347 et note 2] qui écrit: "la conciliation est [...] le mode de droit commun pour la résolution des litiges dans de nombreux pays, Chine et Japon et Etats africains notamment"; (J.) El Hakim, op cit.: "[... dans de nombreux pays (tribus arabes, asiatiques ou africains, Chine, Japon, etc..) la conciliation représente, depuis des millénaires, le mode de règlement des conflits et n'a cessé de coexister avec les processus modernes de règlement et de s'y adapter".

Voir (M.) Landrau, Justice "indigène" et politique coloniale, l'exemple de la Côte d'Ivoire (19031940), Penant 1978, p. 5 et s., voir Spéc., n 8 , p. 13 et note 25.

Voir "La Côte d'Ivoire", Notices publiées par le gouvernement général [de l'Afrique occidentale Française] à l'occasion de l'exposition coloniale de Marseille, éd. Creté, Imprimerie typographique Corbeil, 1906, p. 272 et s. (désormais cité sous Notice...) 
sentence prononcée. Il s'agit de discussions plus ou moins longues où les deux parties écoutent les avis, les uns des autres, cèdent à l'influence du chef de famille, à l'opinion publique. Après de nombreux marchandages, la partie lésée admet la réparation proposée, tandis que l'auteur du dommage présente des excuses et s'engage à payer l'indemnité fixée $^{54}$. S'il s'agit de deux individus de familles différentes, ce sont les deux hommes les plus vieux de chaque famille qui discutent l'indemnité et prennent fait et cause pour les intéressés.

Quand un habitant d'un village a commis un délit dans une autre localité, la cause est appelée devant le chef de village où le délit a été commis; mais le chef de village de la personne en cause intervient et les affaires se règlent entre les deux chefs ${ }^{55}$. Les procédures civile et pénale sont identiques. Une affaire civile et criminelle est introduite devant "le juge", par la plainte de la partie lésée. Le rôle "du juge" est beaucoup plus d'amener une entente entre les parties au sujet de la fixation de la réparation demandée, que de prononcer une sentence. C'est somme toute un tribunal de conciliation chargé de diminuer les demandes exagérées de la partie lésée pour en arriver aux conditions habituelles fixées par la coutume.

Avec l'avènement de la colonisation, les pouvoirs de conciliation des chefs traditionnels vont être maintenus. En effet, en 1903 par un décret en date du 10 novembre seront créés des tribunaux de village, de province et de cercle. Dans chaque village, le législateur colonial institue un tribunal à juge unique: le chef de village est investi d'un pouvoir de conci-

On relèvera que, dans la quasi-totalité des régions du pays, les peines infligées sont basées sur l'idée de dédommagement et non sur celle de châtiment. Les peines corporelles, n'existent pas; la seule peine appliquée est l'amende qui est alors la compensation pécuniaire du dommage causé. Cela s'apparente alors à la transaction.

Il faut préciser à cet égard, que la justice était rendue, notamment dans la région du centre (Baoulé) par des tribunaux ou assemblées de quatre sortes:

- Le conseil de famille, comprenant les membres d'une famille et présidé par son chef; il a à connaître les délits commis par les membres de la famille au préjudice de la famille même, ou de contestations entre deux de ses membres;

- Le conseil de village, comprenant les notables du village et présidé par l'un d'eux; il s'occupe des délits commis par un habitant du village au préjudice d'un autre habitant appartenant à une famille ou membres de différentes familles de ce village;

- Le conseil de tribu, composé de notables de différents villages appartenant à un même groupe ethnique ou politique, et présidé par le plus ancien d'entre eux; il connaît des délits ou affaires civiles intéressant les familles de villages différents d'une même tribu;

- Le Conseil arbitral, composé de quelques notables, présidé par un arbitre, choisi par les deux parties; ce tribunal connaît des délits graves, des règlements d'indemnité de guerre et suspensions d'hostilités ainsi que des affaires auxquelles se trouvent mêlés deux tribus ou deux groupements différents [voir "La Côte d'Ivoire", Notices..., op. cit., p. 471 et s.]. 
liation en matière civile et commerciale pour tout litige que lui soumettraient les parties ${ }^{56}$. On relève une tendance naturelle des administrés à faire régler leurs différends par le chef de village. On préfère s'arranger à l'amiable devant le chef de village qui règle l'affaire en conciliation; si bien que même lorsque le législateur colonial a, un peu plus tard, limité les compétences du tribunal de village, il a conservé la procédure de conciliation ${ }^{57}$. Cette procédure va être abandonnée dans les textes nouveaux pris au lendemain des indépendances en 1960. Le législateur ivoirien adopte des principes universellement reconnus, qui ne font aucune référence au pouvoir de conciliation des autorités traditionnelles. Pourtant, dans les faits, on constate qu'elles continuent d'exercer ce pouvoir sans que pour autant les autorités étatiques ne s'en soucient.

\section{La survivance de la fonction judiciaire des autorités traditionnelles ${ }^{58}$}

Bien qu'il n'existe pas de texte spécifique en la matière ${ }^{59}$, les autorités traditionnelles (notamment les chefs de village et leurs notables) continuent de régler en tant que conciliateurs - les litiges dans les villages. Ce faisant, ces autorités rendent une véritable justice

L'organisation judiciaire mise en place par ce texte repose sur les principes suivants: la justice française réservée presque exclusivement aux français, européens ou assimilés; la justice dite indigène rendue aux administrés selon la coutume notamment au 1er degré.

En 1924, le décret ne mentionne plus l'existence du tribunal; il reconnaît seulement, en matière civile et commerciale, le pouvoir de conciliation du chef de village, conciliation dont, d'ailleurs, il affaiblit la portée; en 1er lieu,, elle aura la force probante d'un acte sous seing privé seulement si elle est constatée par un fonctionnaire Français; en second lieu, le décret confié au tribunal du 1er degré la charge de tenter de concilier les parties en matière civile et commerciale, avant toute instruction ou jugement.

58 Sur la notion d'autorité traditionnelle, voir (O. H.) Legré, Autorité traditionnelle et développement en Afrique, le cas de la Côte d'Ivoire de 1960 à nos jours, in Pouvoirs publics et développement en Afrique, S.E.D.U.S.S. (Service des Editions de l'Université des Sciences Sociales, 1992), p. 167 et s. L'auteur écrit: "Dans la littérature historique, l'expression" autorité traditionnelle "se ramène à l'ensemble des pouvoirs consacrés par les coutumes négro-africaines et entérinés par l'ordre politique étatique colonial ou postcolonial. Cette autorité traditionnelle a par conséquent sa source de légitimité et ses mécanismes de légitimation dans la coutume, mais détient sa légalité de textes législatifs du pouvoir étatique qui consacre en aval les procédures d'investiture en amont. Elle fonctionne donc dans une dualité institutionnelle dont les fondements et les finalités sont diamétralement opposés".

Selon les dispositions de l'article 1er de la loi $\mathrm{n}^{\circ}$ 61-155 du 18 mai 1961 portant organisation judiciaire, "dans la République de Côte d'Ivoire, la justice est rendue en matière civile, commerciale, pénal, l'administrative par la cour suprême, des cours d'appel, par des tribunaux de première instance et des section détachées de ces tribunaux". L'article 5 du code de procédure civile, commerciale et administrative indique quant à lui que: "les tribunaux de première instance et leurs sections détachées, connaissent de toutes les affaires civiles, commerciales, administratives et fiscales..." on relèvera, enfin, que la Constitution ivoirienne, en son article 58, énonce notamment que la justice est rendue sur toute l'étendue du territoire de l'Etat au nom du peuple; et que les juges ne sont soumis, dans l'exercice de leurs fonctions, qu'à l'autorité de la loi. 
informelle ou justice parallèle ${ }^{60}$. Lorsque, par exemple, un débiteur n'arrive pas à s'acquitter de sa dette, son créancier va menacer de le traduire devant la chefferie. Si, malgré cette mise en garde, le débiteur ne s'exécute toujours pas, le créancier va rencontrer seul, les chefs traditionnels et leur exposer le problème. La chefferie va ensuite convoquer les deux parties devant le chef de village; un délai d'un, de deux ou trois mois est alors fixé au débiteur pour qu'il honore sa dette ${ }^{61}$. A la fin de l'échéance fixée - et en cas de non paiement - le créancier va demander au chef "un papier" justifiant que l'affaire est passée devant la chefferie. C'est à partir de ce moment que, muni du document établi par le chef que le créancier va se rendre au poste de gendarmerie. Il y alors mise en mouvement de la procédure officielle ${ }^{62}$.

Peut-on recourir directement à la justice étatique? Il apparaît selon les informations recueillies qu'il n'est pas possible de soumettre directement le litige aux juridictions étatiques. Ainsi, si un habitant du village passant par dessus la chefferie, se rendait directement à la gendarmerie (ou au tribunal) pour régler son problème, il se verra renvoyé par les agents de la gendarmerie à saisir d'abord le chef de village, il y a donc une étroite collaboration entre la gendarmerie et la chefferie. Mieux, il a été donné de constater que pour une affaire déterminée à l'occasion de laquelle le plaignant a saisi directement le juge territorialement compétent, le chef de village a demandé et obtenu que le juge sursoit à statuer en attendant le règlement traditionnel du litige ${ }^{63}$.

Voir à cet égard, en droit togolais, (A.) Mignot, La justice traditionnelle, une justice parallèle, Penant 1982, p. 5 et s.

Une autre porte de sortie est possible: le débiteur peut donner sa plantation en exploitation pour le remboursement de sa dette. On dit que son champ est pris en "hypothèque". Grâce à cette opération, le créancier va utiliser la plantation pendant une période donnée; le temps que celui-ci, par le profit tiré de l'exploitation du champ puisse récupérer la totalité du montant de la somme qu'on lui doit. Bien entendu, des documents (signés par le créancier, le débiteur et le chef) sont établis attestant que la plantation n'est pas illégalement exploitée par le créancier. Ces informations ressortent d'une enquête que nous avons mené à Songon.- Agban, village situé à une vingtaine de kilomètres d'Abidjan.

Cette procédure traditionnelle est pratiquement la même qui est suivie en matière pénale: Si un vol, par exemple, est commis par un enfant; la victime va en informer le chef qui va entendre le prévenu et, si ce dernier reconnaît les faits, on fera appel à ses parents;. Si ceux-ci acceptent d'indemniser la victime, l'affaire prend fin. Dans le cas contraire, on retombe dans la procédure décrite pour les affaires civiles.

L'exemple en cause concerne une plainte déposée par un ressortissant de Songon-Agban (village situé à une vingtaine de kilomètres d'Abidjan) devant la section de tribunal de Dabou. Il nous a été rapporté que cette situation prévaut également dans le village de Bacon-S/P Akoupé (situé à une centaine de kilomètres d'Abidjan): pour tout litige opposant des habitants du village, on passe nécessairement devant le conseil de village présidé par le chef assisté de quatre notables représentant chacun l'un des quatre quartiers de cette bourgade. 
Cette dernière situation paraît quelque peu surprenante. On peut d'ailleurs se demander quelle est la force des décisions des chefs de village. Il faut relever d'abord que les autorités traditionnelles ne peuvent imposer leur médiation: un jugement ne peut avoir lieu que dans la mesure où le défendeur accepte que l'on débatte de l'affaire à propos de laquelle il est assigné. Ensuite, les décisions rendues sont dépourvues de toute force exécutoire: celles-ci ne valent que dans la mesure où les parties consentent à s'y conformer ${ }^{64}$. L'obligation de soumettre le litige aux chefs traditionnels n'est donc, en définitive, qu'une obligation morale.

Quoi qu'il en soit, les autorités officielles (ou étatiques) laissent les chefs coutumiers régler une bonne partie des litiges survenant au sein de leurs communautés car cela permet non seulement de sauvegarder la cohésion sociale, mais également de résorber le problème posé par les insuffisances de la justice étatique. On peut, peut-être, regretter que le législateur ivoirien n'ait pas songé, à l'instar de son homologue nigérien ${ }^{65}$, à institutionnaliser cette pratique. En droit nigérien, le texte de base qui traite du conciliateur traditionnel est l'article 78 de la loi $n^{\circ}$ 62-11 du 16 mars 1962 fixant l'organisation et la compétence des juridictions nigériennes. L'alinéa 1 de ce texte dispose: "... le chef de canton ou de groupement, le chef de village ou de tribu désignés à cet effet par la coutume sont investis du pouvoir de concilier les parties dans les matières coutumières, civiles ainsi que commerciales, susceptibles de transaction".

Le conciliateur, en droit positif nigérien a un rôle facultatif dans le processus de règlement de conflit. Sa conciliation est un préliminaire facultatif de règlement des conflits ${ }^{66}$. En cas d'accord, il est dressé un procès-verbal de conciliation auquel le législateur attache des effets précis. Aux termes de l'article 78 alinéa 5 de la loi du 16 mars 1962 précitée, "l'accord [de conciliation] intervenu acquiert la force probante des actes sous seing privé lorsqu'il a été constaté par écrit. S'il demeure à l'état de convention verbale, il possède la valeur reconnue par la coutume aux conventions de cette nature". Que ce passera-t-il si l'une des parties à l'accord venait à contester celui-ci? Une ordonnance $n^{\circ}$ 93-28 du 30 mars 1993 portant statut de la chefferie traditionnelle en république du Niger permet de résoudre la question. Son article 15 alinéa 3 énonce: "... il [le chef traditionnel] dresse les procès-verbaux de ses conciliations qui doivent être consignés dans un registre ad hoc dont extrait est adressé à l'autorité administrative et à la juridiction compétente". "Les procès-

Voir (A.) Mignot, op cit., $\mathrm{n}^{\circ} 10$, p. 11 et 12 . Pourrait-il en être autrement in présence d'une conciliation? Le fait est qu' «un élément essentiel de la justice est que l'on peut demander et recevoir un règlement définitif et effectivement appliqué à un conflit, si une conciliation privée se révèle impossible»: (U.) Spellenberg, op cit., p. 153 et s., voir Spéc. p. 165.

Voir (A.) Chä̈bou, Il faut noter que le Cameroun a conservé ses juridictions coutumières traditionnelles: voir (P.) Nkou Mvondo, op cit., p. 210, note 7.

66

(A.) Chaïbou, ibid., p. 140. 
verbaux dressés et signés par les parties ne sauraient en aucun cas, être remis en cause devant l'autorité administrative ou judiciaire." Cette construction est intéressante et gagnerait à être étendue à la Côte d'Ivoire. Quoi qu'il en soit, s'il y a de lege lata, absence de texte général dans lequel évolue le conciliateur traditionnel; on note l'émergence, ces dernières années d'un cadre réglementaire spécial.

A cet égard, le décret $n^{\circ}$ 96-433 du 3 juin 1996 relatif au règlement des différends entre les agriculteurs et les éleveurs mérite d'être relevé. Ce texte met en place trois commissions (une commission villageoise de règlement à l'amiable, une commission sous-préfectorale et une commission préfectorale de recours et d'arbitrage ${ }^{67}$ ). L'article ler du décret dispose: "lorsqu'un différend oppose un éleveur et un agriculteur au sujet d'un dégât causé aux cultures par des animaux domestiques ou d'un préjudice subi par un ou plusieurs animaux, les parties doivent rechercher un règlement amiable." ${ }^{68}$ pour ce faire, il est installé, par le sous préfet des commissions villageoises de conciliation. Celles-ci, présidées par les chefs de villages, comprennent en outre un représentant des éleveurs et un représentant des agriculteurs du ou des villages concernés.

Les litiges entre agriculteurs et éleveurs conduisent parfois à des situations sanglantes voir mortelles. C'est pourquoi l'article 4 du décret du 3 juin 1996 précité énonce qu' «en cas d'échec de la commission villageoise dans la recherche de règlement amiable ou si les accords conclus devant la commission villageoise n'ont pas été réalisés dans les huit jours suivant leur adoption, le différend peut être porté devant la commission sous-préfectorale Celle-ci présidée par le sous-préfet comprend en outre deux techniciens des services extérieurs du Ministère chargé de l'agriculture et des ressources animales, le chef de village concerné, un représentant des éleveurs du village concerné, un représentant des agriculteurs du village concerné."

Quelles sont les sanctions prévues et quelle est la force exécutoire des décisions (délibérations) des commissions? L'article 15 du décret dispose: "en cas de non-paiement des sommes dues par un auteur identifié, le sous-préfet utilise toutes les voies de droit pour recouvrer les fonds destinés à la victime, notamment le recours à huissier". Par ailleurs, selon les dispositions de l'article 19 "tout éleveur qui ne se soumet pas aux décisions des commissions $[\ldots]$ et n'exécute pas les sanctions par elles prononcées peut se voir retirer sa carte d'électeur par l'autorité administrative compétente".

La dernière commission composée de personnalités de la circonscription notamment le préfet, les députés et les maires, a, entre autres, pour mission de veiller au bon fonctionnement des commissions sous-préfectorales et de proposer si besoin est la révision de la réglementation en vigueur. 
Il faut donc retenir que le décret du 3 juin 1996 offre un exemple d'une collaboration tant des autorités judiciaires, administratives que traditionnelles pour le règlement global de litiges. Cette collaboration entre autorités officielles et instances officieuses se retrouve, à un degré moindre il est vrai, dans un autre mode négocié de règlement des litiges: la justice de proximité.

\section{La justice de proximite t9 $^{69}$}

L'expression recouvre les modes informels de règlement des litiges dans les quartiers urbains pauvres où le mode de vie traditionnel est encore vivace. Il faut identifier les instances concernées par cette "justice" ainsi que le domaine de leur intervention, de même que les règles qui y sont applicables. Il ressort de l'enquête effectuée par le professeur René Degni-Ségui $^{70}$ que les instances impliquées dans la justice de proximité sont pour l'essentiel et selon les cas, des chefs de quartier et présidents de comités de résidents ${ }^{71}$, des chefs de communautés ethniques ${ }^{72}$, des responsables politiques locaux et des leaders religieux.

Les instances ci-devant citées sont souvent sollicitées pour le règlement d'affaires civiles, commerciales et même pour quelques menues infractions ${ }^{73}$. Les règles applicables par la justice de proximité découlent des valeurs traditionnelles. Celles-ci traduisent la conception africaine de la justice qui tend plus à concilier les deux parties qu'à désigner un gagnant et un perdant ${ }^{74}$. C'est la raison pour laquelle elle a la préférence des justiciables. Cela n'est pas étonnant si l'on sait que près de $70 \%$ de la population ivoirienne demeure profondé-

L'expression est du professeur René Dégni-Ségui. Modes informels de régulation dans les quartiers urbains pauvres., op cit., p. 20 et s.

70

(R.) Degni-Segui, Ibid.

71 Les chefs de quartier et les présidents de comités de résidents sont l'émanation des populations de leur quartier. Contrairement aux présidents de comité de résidents qui sont toujours élus et dont l'existence révèle une certaine organisation des quartiers, les chefs de quartier, eux, ne sont pas forcement élus et tirent leur légitimité de l'histoire même du quartier. De fait, les chefs de quartiers sont généralement les anciens chefs du village devenu aujourd'hui quartier d'une commune d'Abidjan (on peut citer, par exemple, le chef de Blockhaus-quartier de la commune de Cocody). Lorsqu'ils ne sont pas élus, ils sont propriétaires terriens.

Il s'agit de chefs élus par des allogènes ou d'étrangers qui se retrouvent dans une commune ou une ville qui n'est pas celle de leurs origines (ethniques).

Les personnes interrogées dans le cadre de l'enquête menée par le professeur Dégni-Ségui [ article précité] citent expressément les conflits familiaux (problèmes de foyers et d'adultère, coups et blessures, problèmes successoraux, différends entre membres d'une même famille), les conflits de voisinage (coups et blessures entre habitants du même quartier, problèmes de loyer, abus de confiance portant sur de petites sommes...).

Voir (R..) Dégni-Ségui, Ibid., p. 22 
ment attaché aux valeurs traditionnelles ${ }^{75}$. C'est aussi ce qui explique que les autorités tolèrent mais aussi encouragent cette "justice" originale "à en juger de par l'attitude des agents des forces de l'ordre qui y renvoient certaines affaires pour y être préalablement et même définitivement traitées" 76 .

De ce qui précède, il apparaît que le justiciable rejette la justice étatique. Cette attitude va amener les pouvoirs publics à favoriser ou encourager, dans bien des cas, le recours à des procédés traditionnels de résolution de litiges. Par ailleurs, les justiciables, notamment ceux habitant les villes, étant marqués par le processus de modernisation, la voie sera alors ouverte à l'avènement de modes nouveaux de règlement des litiges.

\section{L'avènement de modes nouveaux de règlement des litiges}

En Côte d'Ivoire, comme partout ailleurs en Afrique, la crainte de représailles conduit, presque toujours, le justiciable à s'abstenir de mettre judiciairement en cause l'administration et à rechercher une voie négociée de résolution de son litige. Dans ce sens, la médiation d'une autorité administrative, généralement le Président de la République, sera souvent sollicitée.

Le monde des affaires aussi craint d'avoir à affronter un juge qu'il estime à tort ou à raison, lent, incompétent, partial et corrompu ${ }^{77}$. Aussi évite-t-il souvent de recourir à la machine judiciaire pour en appeler à l'arbitrage. Ces considérations ont suscité, de la part des pouvoirs publics, de récentes interventions qui se traduisent par la consécration de l'arbitrage (A) et l'institutionnalisation de la médiation (B).

\section{A. La consécration de l'arbitrage}

L'arbitrage est un mode juridictionnel de règlement d'un litige par une autorité qui tient son pouvoir de juger, non d'une délégation permanente de l'Etat ou d'une institution internationale, mais de la convention des parties (lesquelles peuvent être de simples particuliers ou des Etats). L'arbitrage est, ainsi, une justice à fondement conventionnel ${ }^{78}$. Dès lors, l'existence de cette procédure de résolution des litiges ne devrait pas, dans un pays comme la Côte d'Ivoire qui a hérité du code Napoléon, poser difficulté. Mais le fait est que

Voir (J.) Assi Benié, op cit., p. 5

76

77 Voir (M.) Debene, op cit. 1990, p. 92.

78

(R.) Perrot, Institutions judiciaires, Montchestien, 7ème éd., 1995, n 61 . 
l'arbitrage peut être ad hoc ou institutionnel ${ }^{79}$. Un problème s'est alors posé dans un passé très récent à propos de l'existence de l'arbitrage commercial en Côte d'Ivoire qui a rendu nécessaire l'intervention de la loi du 9 août 1993.

\section{De l'existence de l'arbitrage commercial}

Lorsqu'en 1972 la Côte d'Ivoire devait adopter son code de procédure (loi n ${ }^{\circ} 72-833$ du 21 décembre 1972 portant code de procédure civile, commerciale et administrative) l'on pouvait, raisonnablement, s'attendre à ce qu'il y soit prévues des dispositions relatives à l'arbitrage commercial. Il n'en a rien été. Cette situation ne manquera pas de susciter des difficultés car les parties recouraient toujours à l'arbitrage: la jurisprudence était divisée quant à l'admission de l'arbitrage commercial en Côte d'Ivoire. La controverse peut être illustrée par deux décisions de la Cour suprême, rendues en 1986 et $1989^{80}$.

De l'analyse de la jurisprudence et des commentaires doctrinaux ${ }^{81}$ faits à propos de celleci, il se posait moins la question de l'admission ou de la reconnaissance de l'arbitrage ${ }^{82}$ que celle de la force exécutoire de la sentence arbitrale. La Cour suprême dans sa décision de 1986 a censuré l'arrêt d'appel qui avait validé une sentence arbitrale. La cassation de la

Voir les définitions données par (G.) Cornu, op cit.: L'arbitrage ad hoc se déroule en dehors de toute organisation permanente d'arbitrage et relève de la seule initiative des parties et de leurs arbitres; tandis que l'arbitrage institutionnel suppose le concours d'un organisme permanent d'arbitrage (p. ex. chambre de commerce international) qui met à la disposition des litants une liste d'arbitres, un règlement d'arbitrage, une organisation matérielle (secrétariat, locaux, etc... et des services (notification des mémoires, p. ex.).

Cour suprême, 29 avril 1986: "attendu que s'il est exact que les parties peuvent insérer dans l'acte qui les lie une clause compromissoire visant à une procédure d'arbitrage, il n'en reste pas moins vrai que les conditions et les modalités de cet arbitrage doivent être prévues par le législateur (...)". Cour suprême, 4 avril 1989: "... s'il est constant que le code de procédure civile, commerciale et administrative n'a ni prévu, ni organisé l'arbitrage, il est non moins constant que pour l'application dudit texte, les juridictions ivoiriennes ont recours soit aux principes généraux du droit, soit aux dispositions du livre III du code de procédure Français à titre de raison écrite; qu'il s'ensuit que la cour d'appel, en déclarant valables la clause compromissoire et la sentence qui en résulte n'a aucunement violé les textes visés en moyen".

81 (L.) Idot, La Cour suprême et la réglementation de l'arbitrage en Côte d'Ivoire: A propos de l'arrêt du 29 avril 1986, Revue Ivoirienne de Droit Economique et comptable (RIDEC), mars 1987, $\mathrm{n}^{\circ} 1$ p. 3 et s. Dès l'entame de son article, l'auteur s'interrogeait sur la question de savoir si l'on peut "recourir à 'arbitrage comme mode de règlement des différends commerciaux en Côte d'Ivoire"; Eléments d'un droit international privé africain: L'exemple de la Côte d'Ivoire, Revue Juridique Africaine, $\mathrm{n}^{\circ}$ 1, 1990, p.7 et s., voir Spéc., p. 32 et s. (H.) Meledje, La question arbitrale et la fonction juridictionnelle en Côte d'Ivoire, Annales de l'Université de Cocody, série A, Droit, Tome X, 1995, Abidjan, p. 9 et s.

Cela n'est pas étonnant puisque l'arbitrage (qui peut être $a d$ hoc) est un procédé conventionnel de règlement des litiges. Il résulte précisément d'une convention d'arbitrage renfermant une clause compromissoire ou un compromis. 
décision des juges du fond reposait sur les motifs suivants: "(...) s'il est exact que les parties peuvent insérer dans l'acte qui les lie une clause compromissoire visant à une procédure d'arbitrage, il n'en reste pas moins vrai que les conditions et les modalités de cet arbitrage doivent être prévues par le législateur". Cette motivation, est selon le professeur Idot, pour le moins surprenante: les clauses compromissoires (ou les compromis) sont valables mais l'arbitrage est inefficace faute de réglementation étatique en la matière, car la sentence arbitrale ne peut être validée. A quoi sert d'admettre, poursuit plus loin l'auteur, la validité de la clause compromissoire en matière commerciale, si l'exécution de la sentence est refusée aux parties? Le professeur Idot en a alors déduit qu'il y avait exclusion de l'arbitrage commercial tant au plan interne qu'international en Côte d'Ivoire.

Cette position n'est pas partagée par M. Meledje dont la thèse se présente ainsi: "(...) le principe de l'arbitrage commercial tant en droit interne qu'en droit international est admis en Côte d'Ivoire et (...) une sentence exécutoire peut valablement résulter d'une juridiction arbitrale qui, sur ce plan, est autosuffisante et ne nécessite pas la force exécutoire que confère l'exequatur éventuel du juge étatique pour être effectif" ${ }^{\prime 83}$. Nous estimons, quant à nous, que l'une et l'autre opinion auraient gagné à être nuancée: s'il est vrai que l'arbitrage commercial était admis en Côte d'Ivoire, il s'agissait bien d'un arbitrage "handicapé". En effet, le refus de l'exequatur prive bien l'arbitrage de l'un de ses effets ${ }^{84}$. Et si l'exequatur, c'est à dire l'exécution forcée, ne peut être demandée que dans l'hypothèse d'une contestation, cette situation pour être éventuelle n'en est pas moins possible.

Dès lors, une autre question se posait: le juge ivoirien est-il fondé à octroyer la force exécutoire à une sentence arbitrale nationale ou internationale indépendamment de toute autorisation du législateur? C'est ainsi, finalement, la question à laquelle la Cour suprême a répondu d'abord négativement dans sa décision de 1986, puis par l'affirmative dans celle de 1989. C'est aussi la question qui a amené Madame le professeur Idot à écrire: "en définitive, le droit ivoirien de l'arbitrage apparaît bien incertain et surtout très lacunaire. (...) Il est urgent que le législateur intervienne pour combler les nombreux vides" ${ }^{85}$. Ce plaidoyer aura été entendu par le législateur ivoirien.

(H.) Meledje, Ibid., p. 11; voir aussi p. 16, note 18 où l'auteur fait la distinction entre décision exécutoire et décision revêtue de la force exécutoire: "la première, au contraire de la seconde, ne peut faire l'objet d'une exécution forcée par le biais des forces publiques. Ce qui ne veut pas dire qu'elle ne peut être exécutée volontairement dès lors que son caractère définitif est avéré et qu'elle émane bien d'une juridiction".

Voir sur ce point: (L.) Cadiet, Droit judiciaire privé op cit. n 2047 et s. L'auteur distingue, en ce qui concerne l'efficacité de la sentence arbitrale, les effets indépendants de l'exequatur de ceux qui lui sont subordonnés.

(L.) Idot, La Cour suprême et la réglementation de l'arbitrage en Côte d'Ivoire, op cit.; voir aussi Eléments d'un droit international privé africain, op cit. 
L'Etat ivoirien a toujours été favorable à l'arbitrage comme mode de règlement des litiges en matière internationale, qu'il s'agisse de contrats d'Etat ou des contrats entre personnes privées ${ }^{86}$. Il était donc inconcevable que la Côte d'Ivoire n'ait pas sa propre réglementation de l'arbitrage. Le 9 août 1993, le législateur ivoirien va remédier à cette lacune. Dans le courant de la même année sera signé, entre plusieurs pays africains de la Zone franc dont la Côte d'Ivoire, le 17 octobre à Port-Louis, le traité OHADA (Organisation pour l'harmonisation en Afrique du Droit des Affaires) ${ }^{87}$. Ce traité organise aussi une procédure d'arbitrage.

\section{a) La loi du 9 août 1993 relative à l'arbitrage ${ }^{88}$}

L'intervention de cette loi, dont le contenu doit être précisé, va susciter la création d'un organisme permanent d'arbitrage au sein de la chambre de commerce et d'industrie de Côte d'Ivoire. Le texte, qui s'applique à l'arbitrage national et international ${ }^{89}$, aborde la plupart des questions essentielles relatives à la matière. Parmi celles-ci, et dans le cadre de cette étude, seules celles concernant la convention d'arbitrage et l'exécution des sentences méritent qu'on s'y arrête. Relativement à la convention d'arbitrage, trois points sont retenus: sa forme, son domaine (le problème de l'arbitrabilité) et ses effets. La convention d'arbitrage revêt les deux formes classiques à savoir la clause compromissoire qui ne peut être usitée qu'en matière commerciale (article ler de la loi) et le compromis (article 6).

L'arbitrabilité recouvre deux formes qui posent autant de questions: L'arbitrabilité subjective: tout organisme peut-il recourir à l'arbitrage? Plus précisément, l'Etat peut-il compromettre? Il résulte de l'article 17 de la loi que l'Etat ne peut compromettre ${ }^{90}$.

Comme en témoigne son adhésion aux conventions suivantes:

- Convention pour la reconnaissance et l'exécution des sentences arbitrales étrangères, en date, à New York, du 10 juin 1958 (ratifiée par la Côte d'Ivoire le 1er février 1991).

- Convention de Washington du 18 mars 1965 sur le règlement des différends relatifs aux investissements entre Etats et ressortissants d'autres Etats (signée par la C.I. le 30 juin 1965; dépôt des instruments de ratification le 16 février 1966; entrée en vigueur de la convention le 14 oct. 1966).

L'OHADA est une organisation supranationale chargée d'uniformiser le droit des affaires par le moyen d'actes uniformes qui s'imposent directement dans le droit interne des Etats parties. L'un des actes uniformes traite de l'arbitrage.

89 Loi N 93-671 du 9 Août 1993 (J.O.R.C.I. du 16 Septembre 1993, p. 675 et s.).

Aux termes de l'article 50, "est international l'arbitrage qui met en cause les intérêts du commerce international".

Selon le deuxième alinéa de cette disposition, seules "des catégories d'établissements publics à caractère industriel et commercial peuvent être autorisées par décret à compromettre". On peut se demander si ce texte s'applique à l'arbitrage international. 
L'arbitrabilité objective: peut-on soumettre à l'arbitrage tout litige? En d'autres termes, tel ou tel litige (matière) peut-il être confié à des juridictions étatiques uniquement? l'article 17 alinéa $1^{\text {er }}$ précise qu'il n'est pas possible de compromettre ni sur les questions d'état, de capacité des personnes, de divorce et de séparation de corps; ni sur les contestations intéressant les collectivités publiques et les établissements publics; ni plus généralement dans les matières qui intéressent l'ordre public. Les effets de la convention se dédoublent: il y a un effet négatif et un effet positif.

L'effet négatif consiste en ce que, en présence d'une clause d'arbitrage, les tribunaux étatiques doivent se déclarer incompétents (article 16 de la loi). Toutefois, il faut que le défendeur soulève l'exception d'incompétence sur le fondement de la clause compromissoire. Par conséquent, le juge ne peut soulever d'office son incompétence au motif qu'il existe une clause d'arbitrage ; il doit se reconnaître compétent.

L'effet positif réside dans ce que les parties ont l'obligation de soumettre leur litige à une procédure d'arbitrage; le défendeur, notamment, est obligé d'exécuter la convention d'arbitrage ${ }^{91}$.

Quant à l'exécution des sentences arbitrales ; Les effets classiques reconnus aux décisions arbitrales y sont admis: La sentence arbitrale a, dès qu'elle est rendue, l'autorité de la chose jugée relativement à la contestation qu'elle tranche (article 34); la décision de l'arbitre n'étant susceptible d'exécution forcée qu'en vertu d'une ordonnance d'exequatur (article 35 de la loi). Ces effets s'appliquent également à l'arbitrage international (article 58).

De la mise en œuvre de cette loi, on aboutit inéluctablement à la création de la Cour d'Arbitrage de Côte d'Ivoire (CACI). Installée officiellement le 12 août 1997, la CACI est en réalité un centre d'arbitrage qui a pour fonction d'organiser et de superviser, conformément à son règlement, les arbitrages effectués sous son égide.Elle a pour mission d'offrir aux opérateurs économiques des moyens de conciliation et d'arbitrage pour le règlement de leurs différends ${ }^{92}$. Quelles sont les raisons qui ont milité en faveur de la création de la

Voir, sur ce point, (J. M.) Mousseron [op cit., $\mathrm{n}^{\circ}$ 1464] de qui on apprend que la clause compromissoire crée à la charge des parties une triple obligation: ne pas saisir le juge qui au cas contraire, devrait décliner sa compétence; compromettre en organisant de façon particulière l'arbitrage du différend qui les oppose; respecter la sentence arbitrale dont, en cas de méconnaissance, le juge imposera, en dehors d'un contrôle au fond, le respect par la procédure d'exequatur.

92 Article 1er du règlement d'arbitrage de la CACI: "Il est créé par la chambre de commerce et d'industrie de Côte d'Ivoire (...) la cour d'arbitrage de Côte d'Ivoire dite CACI ayant pour mission d'organiser, (...), la résolution par voie d'arbitrage des différends nationaux et internationaux en matière commerciale...". Article 1er du règlement de conciliations de la CACI: "Tout différend peut faire l'objet d'une conciliation par les soins d'un conciliateur unique choisi d'un commun accord par les parties, ou à défaut désigné par le secrétariat de la CACI". 
CACI? La mise en place de la CACI, qui instaure un arbitrage institutionnel, participe de la crédibilité économique et juridique de la Côte d'Ivoire. De fait, compte tenu du volume sans cesse croissant des différends commerciaux et des connaissances techniques spécifiques qu'exige parfois leur règlement, l'arbitrage commercial s'impose comme un complément ou un substitut nécessaire de la justice étatique. Les opérateurs économiques nationaux et les investisseurs étrangers éprouvaient le besoin d'avoir à leur disposition, hormis la justice étatique, une diversité d'outils et de choix de modes de règlement de leurs différends. En créant la Cour d'arbitrage de Côte d'Ivoire, la Chambre de Commerce et d'Industrie entendait apporter sa contribution à l'assainissement et à la sécurisation de l'environnement juridique des affaires en Côte d'Ivoire ${ }^{93}$. Ce dernier but est l'un de ceux qui ont suscité la naissance des textes de l'OHADA.

\section{b) L'arbitrage de la Cour Commune de Justice et d'Arbitrage de l'OHADA}

Selon le préambule du traité relatif à l'harmonisation du droit des affaires en Afrique, les parties contractantes ambitionnent de promouvoir l'arbitrage comme instrument de règlement des litiges contractuels. Cette tâche est dévolue à la Cour Commune de Justice et d'Arbitrage (C.C.J.A.) ${ }^{94}$. En quoi consiste l'arbitrage de cette juridiction dite de justice et d'arbitrage? En dépit de son appellation, la CCJA ne tranche pas les litiges en tant qu'arbitre. Son intervention, en la matière, est limitée à nommer ou confirmer les arbitres, à être informée du déroulement de l'instance, et à examiner les projets de sentences (articles 21 à 26 du traité).

Quelle que soit la forme de la convention(clause compromissoire ou compromis), toute partie à un contrat peut soumettre un litige d'ordre contractuel ${ }^{95}$ à la procédure d'arbitrage prévue par le traité OHADA ${ }^{96}$; tout tribunal d'un Etat partie saisi d'un litige que les parties étaient convenues de soumettre à un arbitrage doit se déclarer incompétent si l'une des

Voir règlement d'arbitrage et de conciliation de la CACI, voir préface.

94

La CCJA est l'une des institutions de l'OHADA. Elle est juge du contentieux sur l'interprétation et l'application du traité et des actes uniformes. Deux voies de règlement sont prévues pour le contentieux des actes uniformes: la voie judiciaire et la voie de l'arbitrage.

On conviendra avec le professeur (J.) Issa-Sayegh (L'intégration juridique des Etats dans la Zone Franc, Penant, $\mathrm{n}^{\circ} 24,1997$, p. 125 et s., voir $\mathrm{n}^{\circ} 24$, note 163 , p. 150) que tout litige d'ordre contractuel peut être soumis à la procédure d'arbitrage prévue par l'OHADA "même s'il ne s'agit pas de droit des affaires uniformisé par cette organisation, l'article 23 ne donnant aucune précision à ce sujet".

Il s'agit d'une simple faculté, ainsi les parties peuvent, tout à fait valablement, suivre la procédure d'arbitrage nationale qu'elles ont choisie: voir (J.) Issa-Sayegh, Ibid. 164. 
parties le demande, et renverra, le cas échéant, à la procédure d'arbitrage ainsi prévue $\left(\right.$ article 23) ${ }^{97}$.

La faculté de saisir la CCJA est accordée à toute partie qui a son domicile ou sa résidence habituelle dans un des Etats parties ou dont le contrat est exécuté ou à exécuter en tout ou partie sur le territoire d'un ou plusieurs Etats parties (article 21) ${ }^{98}$.

Les sentences arbitrales rendues conformément à la procédure d'arbitrage de l'OHADA ont l'autorité définitive de la chose jugée sur le territoire de chaque Etat partie au même titre que les décisions rendues par les juridictions de cet Etat (article 25 alinéa 1). Elles peuvent faire l'objet d'une exécution forcée en vertu d'une décision d'exequatur que la Cour Commune de Justice et d'Arbitrage est seule compétente à rendre (article 25 alinéa 2 et 3). Cet effet de l'arbitrage s'explique par le fait que l'arbitre qui tranche un litige, a une mission juridictionnelle. Ce qui n'est pas le cas du médiateur dont les propositions doivent être acceptées par les parties.

\section{B. L'institutionnalisation de la médiation}

La médiation est généralement définie comme le mode de solution des litiges consistant, pour la personne choisie par les antagonistes(en raison le plus souvent de son autorité personnelle),à proposer à ceux-ci un projet de solution, sans se borner à s'efforcer de les rapprocher, à la différence de la conciliation ${ }^{99}$, mais sans être investi du pouvoir de le leur imposer comme décision juridictionnelle à la différence de l'arbitrage et de la juridiction étatique $^{100}$. Lorsqu'elle est extrajudiciaire (conventionnelle), la médiation peut être purement conventionnelle (on dit aussi ad hoc) ou institutionnelle.

Aucune précision n'étant portée sur la nature du tribunal, on admettra qu'il peut s'agir d'une juridiction étatique ou d'une juridiction arbitrale. Cette disposition pourrait donc mettre en péril l'avenir de l'arbitrage de la Cour d'arbitrage de Côte d'ivoire dans la mesure où elle se verra privée de nombreux litiges.

Cette disposition, relève, fort justement, le professeur (J.) Issa-Sayegh, "ne manquera pas de poser problème si l'une des parties est domiciliée ou réside dans un Etat partie alors que le contrat est formé et s'exécute dans un Etat hors du champ OHADA ou encore lorsque le contrat s'exécute sur le territoire d'un Etat partie et d'un autre Etat hors du champ OHADA "Il est douteux, dans ces cas, poursuit l'éminent professeur, "que la volonté d'une seule des parties au contrat suffise à déclencher l'application de la procédure d'arbitrage prévue par le traité OHADA": voir article précité, note 166 , p. 150.

99 Sur la distinction médiation / conciliation, voir supra p.11.

100 (G.) Cornu, Vocabulaire juridique, op cit., p. 527. 
Pays de tradition juridique française, la Côte d'Ivoire, qui a hérité du code Napoléon dont la plupart des dispositions y sont encore applicables, connaît depuis toujours la médiation conventionnelle $^{101}$. Celle-ci peut résulter, notamment, de l'insertion, dans le contrat, d'une clause de médiation qui impose alors le recours au médiateur avant la saisine d'une autorité extérieure, judiciaire ou arbitrale ${ }^{102}$.

Toutefois, ces règles de droit dit moderne sont bien souvent méconnues par les justiciables qui préfèrent, cela étant favorisé par la nature du système politique ivoirien, s'en remettre à la médiation du Président de la République. Cette situation est certainement à la base de l'institutionnalisation récente de la médiation par la création de l'Organe Présidentiel de Médiation (O.P.R.E.M.) ${ }^{103}$.

A partir des différents textes qui réglementent cette institution nouvelle, on note, une fois de plus, la volonté des pouvoirs publics de réagir à la crise de la justice étatique. Cela se perçoit à travers la compétence de l'OPREM, l'ouverture de la procédure et son issue.

\section{Un large domaine de compétence}

L'OPREM dirigé par un président, appelé Grand Médiateur ${ }^{104}$, est investi d'un large domaine de compétence dans la mesure où il peut être sollicité à l'occasion de différends et litiges de toute nature. Il est, en effet compétent pour régler aussi bien les différends entre l'administration et les administrés, ainsi que ceux opposant les administrés entre eux. Il peut également être sollicité pour les litiges opposant des communautés urbaines, villa-

101

On a généralement recours à la médiation en matière civile. Cette dernière notion étant ici entendue au sens large, mais il n'est pas exclu qu'on en use dans un domaine aussi hermétique que le droit pénal: voir à cet égard, la transaction en matière pénale (supra); la médiation, tout comme la conciliation, pouvant déboucher sur une transaction. Sur la médiation pénale en général, voir: (G.) Blanc, La médiation pénale, J.C.P. 1994, I, 3760; (M.-C.) Desdevises, Les fondements de la médiation pénale, in Mélanges en l'honneur de Henry Blaise, Economica, 1995, p. 175 et s; (M.-E.) Cartier, les modes alternatifs de règlement des conflits en matière pénale, revue générale des procédures, $\mathrm{n}^{\circ} 1$ janv. / mars 1998, p. 1 et s.; et surtout (Ch.) Lazerges, op cit., p. 217 et s.

102

103

Sur la clause de médiation, voir (J.M.) Mousseron, op cit., $\mathrm{n}^{\circ} 1462$ et 1463.

L'OPREM a été créé par le décret n 95-816 du 29 septembre 1995 (J.O.R.C.I. du 5 octobre 1995, p. 834). On peut regretter qu'une loi (au sens strict du terme) ne soit pas à la base de la création de cette institution. Celle-ci y aurait plus gagné en indépendance. A cet égard, selon M. (J.) Assi Benié (Le médiateur ivoirien, op cit. P. 8) qui relève l'originalité du médiateur ivoirien, "c'est la dépendance du médiateur et non son indépendance vis à vis du président qui devrait lui assurer ses chances de succès" car il se présente en fait comme un allié du pouvoir administratif et précisément du président de la république.

Le Grand Médiateur est nommé par décret du Président de la République. L'actuel Grand Médiateur est M. Mathieu Ekra (voir décret n 96-PR / 12 du 13 Août 1996). Le Grand Médiateur est assisté de médiateurs régionaux. 
geoises ou tout autre entité ${ }^{105}$. Le médiateur ivoirien n'a donc pas, à l'instar de l'ombudsman suédois ou du médiateur français, que des attributions administratives ${ }^{106}$. Il peut intervenir également en matière civile, commerciale, sociale, pénale...

Comment expliquer que le champ de compétence de l'OPREM soit si étendu? La réponse à cette question est fournie par M. Assi Bénié ${ }^{107}$ qui affirme que "l'institution du médiateur apparaît (...) comme l'officialisation d'une pratique, celle du dialogue à l'ivoirienne. Elle est sans doute la réponse du chef de l'Etat à l'inadéquation de l'appareil judiciaire au contexte socio culturel ivoirien".

Cette réponse appelle une autre interrogation: "N'y aura-t-il pas un véritable engouement pour la voie de la médiation d'Etat et une désaffectation corrélative plus accrue à l'égard du prétoire du juge? "Cette question posée par M. Assi Bénié ${ }^{108}$ est en train de trouver réponse ; En effet, on constate, en se référant aux affaires traitées par l'OPREM au cours de l'année 1997, que cette institution connaît un franc succès ${ }^{109}$. Cet empressement des ivoiriens à solliciter l'intervention de cette nouvelle institution peut s'expliquer par les conditions d'ouverture de la procédure.

\section{L'ouverture de la procédure devant l'OPREM}

L'ouverture de la procédure implique ici les modalités de la saisine de l'OPREM ainsi que les effets de cette saisine.

105

106 En France, une

En France, une loi du 3 janvier 1973 a créé un médiateur de la république inspiré du droit Suédois: l'ombudsman créé dans les années 1930. Il est chargé de régler les conflits pouvant exister entre les administrés et les collectivités territoriales, les établissement publics ou les organismes assurant un service public.

(J.) Assi Benié, op cit., p. 8; voir également en ce sens, (R.) Degni-Ségui, L'accès à la justice et 108 ses obstacles, op cit., p. 248 et s.

(J.) Assi Benié, Ibid., p. 9.

109

De la date de nomination du Grand Médiateur (13 août 1996) au 31 décembre 1996, déjà, l'OPREM avait été saisi de cinquante (50) réclamations, alors qu'on connaissait à peine l'existence et l'objet de l'institution. Les affaires traitées pendant cette brève période portent aussi bien sur les requêtes des locataires de logements économiques, les réclamations des droits de licenciement et de pensions de retraite, que sur des litiges à caractère politique et coutumier. Le nombre de réclamations reçues à l'OPREM en 1997 est le double de celui de 1996. En effet, du 1er janvier au 31 décembre 1997, le nombre de requêtes nouvelles s'élève à quatre-vingt dix neuf (99), portant à 149 le nombre total des dossiers effectivement pris en charge et enrôlés comme réclamations. (Source: Rapport au Président de la République des activités de l'OPREM pour 1997). 
Les modes de saisine de l'OPREM sont dépourvus de tout formalisme. Selon la réglementation en vigueur, en effet, l'OPREM est saisi par tout moyen, directement ou indirectement, par l'une des parties en conflit ${ }^{110}$. Dans la pratique, la médiation de l'institution sera sollicitée par le biais d'une simple correspondance.

Lorsqu'il est saisi, le Grand Médiateur apprécie la recevabilité des demandes eu égard aux compétences reconnues aux diverses institutions de l'Etat et de l'état des procédures existantes. Cette dernière précision se conçoit aisément car l'OPREM ne saurait connaître de n'importe quel litige. Il ne pourrait, notamment, être compétent pour intervenir dans une affaire portant sur des droits dont les parties n'ont pas la libre disposition, qui relève du domaine du juge (étatique). C'est ainsi qu'il peut classer provisoirement ou définitivement les demandes et faire connaître sa décision, sommairement motivée, aux parties en litige. Ses décisions sont alors sans recours.

\section{b) Les effets de la saisine}

Selon les dispositions de l'article 21 alinéa 1 du décret du 29 mai 1997 portant règlement de l'OPREM "les recours adressés au grand médiateur emportent suspension par les parties à toutes autres formes de procédure judiciaires ou administratives"

Cette solution signifie qu'une partie ne peut saisir simultanément, par exemple, une juridiction étatique et le grand médiateur. Cette règle est tout à fait logique car en recourant à la médiation de l'OPREM, on a manifesté sa volonté de soustraire, au moins provisoirement, la connaissance de son litige par les autorités judiciaires ou administratives.

Au demeurant, les clauses contractuelles tendant à écarter le traitement judiciaire des litiges ${ }^{111}$ conduisent au même effet.

110

Article 5 du décret du 29 septembre 1995 portant création, organisation et fonctionnement de l'OPREM ; article 16 alinéa 2 du décret du 29 mai portant règlement de l'OPREM. Voir, à titre comparatif, la solution en droit Français: les administrés ne peuvent saisir directement le médiateur de la république. Ils doivent déposer auprès d'un parlementaire de leur circonscription un dossier à remettre au médiateur.

Il s'agit des clauses tendant à favoriser la transaction et de celles tendant à favoriser l'arbitrage. Parmi les premières, on distingue la clause d'arrangement amiable et la clause de médiation. Sur l'ensemble de ces clauses, voir (J.-M.) Mousseron, op cit., $\mathrm{n}^{\circ} 1453$ et $\mathrm{s}$. 
Il faut analyser, ici, les solutions de la médiation et la force intrinsèque des décisions du Grand Médiateur.

\section{a) Les solutions de la médiation}

En cas de succès de la médiation: un procès-verbal, constatant qu'un accord a pu être obtenu entre les parties, est dressé ${ }^{112}$. En cas d'échec de la médiation: Lorsqu'il se trouve dans l'impossibilité d'amener les parties à un accord ${ }^{113}$, le grand médiateur dresse un procès-verbal constatant l'échec de sa mission. Dans cette dernière hypothèse, l'article 19 du décret du 29 mai 1997 précise, en son deuxième alinéa, que les demandeurs "pourront recourir à toute autre procédure de contestation prévue par la loi". La solution contraire aurait été incompréhensible voire inconcevable puisque, non seulement le litige ne reçoit pas d'issue, mais encore le médiateur ne tranche pas, à proprement parler, les différends.

\section{b) La force intrinsèque des décisions du Grand Médiateur}

Les questions classiques concernant les effets du jugement se retrouvent, ici, posées: les décisions du Grand Médiateur ont-elles autorité de chose jugée? ont-elles force exécutoire? Relativement à l'autorité de chose jugé; l'article 7 alinéa 3 du décret du 29 septembre 1995 dispose que "les procès-verbaux dûment signés par les parties en cause, valent renonciation à toute action judiciaire portant sur le même objet et entre les mêmes parties". Cette disposition semble bien indiquer que les décisions de l'OPREM ont l'autorité de chose jugée. Toutefois une petite réserve s'impose: pour que l'autorité de chose jugée puisse être invoquée, il faut qu'il y ait identité d'objet, identité de cause et identité de parties; or la disposition suscitée ne fait cas que de l'objet et des parties. Sans doute s'agit-il d'une mauvaise rédaction du texte qui fait passer l'expression "parties en cause" pour l'identité de cause. Quoiqu'il en soit, l'article 18 du décret du 29 mai 1997 permet, en son deuxième alinéa, de savoir qu'il y a bien autorité de chose jugée puisqu'il énonce "en cas d'accord partiel, les points concernés ne peuvent être autrement contestés par une quelconque voie". Ce qui traduit bien que la décision du médiateur a force de vérité légale.

Le texte (article 19 alinéa 1 du décret du 29 mai 1997) parle d'amiable composition. Quel est en l'espèce le contenu de cette notion? S'agit-il de la mission conférée à l'arbitre de statuer en tant qu'aimable compositeur; pouvoir qui lui permet de trancher le litige en équité, ex aequo et bono, sans être tenu de suivre, sauf si elles sont d'ordre public, les règles du droit? la réponse à cette question est fournie par l'article 5 du décret du 29 septembre 1995 qui indique que "l'Organe Présidentiel de Médiation statue selon l'équité, le bon sens, les coutumes, les usages et les bonnes mœurs". 
S'agissant de la force exécutoire, l'article 21 alinéa 2 du décret, précité, de 1997 dispose: "Les décisions du Grand Médiateur ont force de sentence arbitrale opposable aux parties". On sait que les sentences arbitrales sont exécutoires, mais qu'elles ne sont pas revêtues de la force exécutoire ${ }^{114}$. Par conséquent, si la décision du Grand Médiateur venait à être contestée, par la suite, par l'une des parties, l'autre pourrait alors saisir le juge pour en obtenir l'exécution forcée (par le biais de l'exequatur?). Cette solution accorde à la médiation de l'OPREM une place privilégiée par rapport aux autres modes extrajudiciaires de règlement des litiges.

L'engouement des citoyens pour les modes extrajudiciaires de règlement des litiges tient aux défauts imputés à la justice étatique. Or, c'est par analogie aux tribunaux de l'Etat que se caractérisent les phénomènes de justice non étatique ${ }^{115}$. L'encadrement législatif ou réglementaire devrait, par conséquent, être limité en la matière. Il convient de se souvenir que ces modes conventionnels de règlement des litiges sont caractérisés par la liberté de la volonté de ceux qui y ont recours ${ }^{116}$.

Au demeurant, si à l'ordre social imposé, il faut préférer, pour la préservation de la cohésion sociale, l'ordre social négocié, c'est à la condition de ne pas, forcément, chercher à formaliser l'informel. "En privilégiant la négociation et en orientant le justiciable vers la recherche d'un consensus qui ne se soucie pas de dire le droit mais de concilier les points de vue et les intérêts, les sociétés inventent un nouveau type de droit qui n'a pas besoin de s'exprimer dans la forme canonique du code juridique et d'être énoncé par une instance législative" $^{117}$. Ceci peut être entendu, en fait, comme une nouvelle manière de dire le droit.

115 (J.) Carbonnier, Flexible droit, 8è éd., L.G.D.J., 1995, p. 395.

116 Cette idée doit être nuancée à propos de l'arbitrage qui a une nature mixte: conventionnel par son 117 origine, il est juridictionnel par la fonction qu'exerce l'arbitre.

(E.) Leroy, op cit., p. 118. 